Dementia

and Geriatric

Cognitive Disorders
Dement Geriatr Cogn Disord 2017;43:269-280

DOI: 10.1159/000471507

Accepted: March 15, 2017

Published online: April 28, 2017
(C) 2017 S. Karger AG, Basel

www.karger.com/dem

\title{
Efficacy, Safety, and Tolerability of Armodafinil Therapy for Hypersomnia Associated with Dementia with Lewy Bodies: A Pilot Study
}

\author{
Maria I. Lapid ${ }^{a} \quad K^{2}$ aren M. Kuntz ${ }^{b}$ Sara S. Mason ${ }^{b} \quad$ Jeremiah A. Aakre ${ }^{c}$ \\ Emily S. Lundt ${ }^{c}$ Walter Kremers ${ }^{c}$ Laura A. Allen ${ }^{b}$ Daniel A. Drubach ${ }^{b}$ \\ Bradley F. Boeve ${ }^{b}$ \\ Departments of a Psychiatry and Psychology and ${ }^{b}$ Neurology and ${ }^{\mathrm{c}}$ Division of Biomedical \\ Statistics and Informatics, Mayo Clinic, Rochester, MN, USA
}

\section{Keywords}

Caregivers · Excessive sleepiness · Lewy bodies · Quality of life · Treatment .

Wake-promoting agents

\begin{abstract}
Background/Aims: Hypersomnia is common in dementia with Lewy bodies (DLB). We assessed the efficacy, safety, and tolerability of armodafinil for hypersomnia associated with DLB. Methods: We performed a 12-week pilot trial of armodafinil therapy (125-250 mg orally daily) in DLB outpatients with hypersomnia. The patients underwent neurologic examinations, a neuropsychological battery, laboratory testing, electrocardiography, and polysomnography. Efficacy was assessed at 2, 4, 8, and 12 weeks. Safety assessment included laboratory examinations, QTc interval, and heart rate. Tolerability was assessed by analysis of adverse events. Data were analyzed using the last-observation-carried-forward method. $\boldsymbol{R e}$ sults: Of 20 participants, 17 completed the protocol. The median age was 72 years, most of the participants were men (80\%), and most had spouses as caregivers. The Epworth Sleepiness Scale $(p<0.001)$, Maintenance of Wakefulness Test $(p=0.003)$, and Clinical Global Impression of Change $(p<0.001)$ scores improved at week 12. The Neuropsychiatric Inventory total score
\end{abstract}

ClinicalTrials.gov identifier: NCT01023672. This study was presented in part at the annual meeting of the Alzheimer's \& Parkinson's Diseases Congress - AD/PD 2013, Florence, Italy, March 6-10, 2013; at the International Dementia with Lewy Bodies Conference, Fort Lauderdale, FL, USA, December 1-4, 2015; and the American Academy of Neurology, Vancouver, BC, Canada, April 16-21, 2016.

Maria I. Lapid, MD

Department of Psychiatry and Psychology, Mayo Clinic

200 First St. SW

Rochester, MN 55905 (USA)

E-Mail lapid.maria@ mayo.edu 
( $p=0.003)$, visual hallucinations $(p=0.003)$, and agitation $(p=0.02)$ improved at week 4 . Caregiver overall quality of life improved at week $12(p=0.004)$. No adverse events occurred. Conclusion: These pilot data suggest improvements in hypersomnia and wakefulness and reasonable safety and tolerability of armodafinil therapy in hypersomnolent patients with DLB. Our findings inform the use of pharmacologic strategies for managing hypersomnolence in these patients.

(c) 2017 S. Karger AG, Basel

\section{Introduction}

Dementia with Lewy bodies (DLB) is the second most common cause of degenerative dementia and is estimated to affect approximately 1 million Americans [1-7]. Dementia is the core functional and cognitive manifestation of DLB. Although a systematic review of 10 randomized, placebo-controlled, double-blind trials of cholinesterase inhibitors and memantine showed cholinesterase inhibitors to enhance cognitive function for patients with DLB [8], many other symptoms and features of DLB are not affected by this class of agents. Excessive daytime somnolence is increasingly being recognized in patients with DLB $[3,4,6,9]$. Analyses conducted using the Epworth Sleepiness Scale (ESS), a subjective measure of sleepiness, and the Multiple Sleep Latency Test, an objective measure of sleepiness, in patients with DLB have shown moderate-to-severe sleepiness using both measures [10]. REM sleep behavior disorder, a parasomnia in which patients appear to act out their dreams, is common in patients with narcolepsy and is also common in DLB; this disorder most likely reflects the normal muscle activity of wakefulness invading into REM sleep [11-14]. Visual hallucinations, which are also common in DLB and narcolepsy, could reflect intrusions of dream imagery into wakefulness [11-14].

The use of psychostimulants or the wake-promoting agents modafinil and armodafinil often results in improvements in cognition, excessive daytime sleepiness, and visual hallucinations in patients with narcolepsy [15-18]. We, therefore, conducted an open-label, 12-week study using armodafinil - a wake-promoting agent that has slightly more potency and duration of effect than modafinil - to determine its efficacy, safety, and tolerability in patients with DLB. We hypothesized that armodafinil would show efficacy on measures of excessive daytime sleepiness, impaired functional status, cognitive impairment, neuropsychiatric morbidity, and patient/caregiver quality of life (QOL), as well as be safe and well tolerated.

\section{Subjects and Methods}

\section{Patients}

From January 2010 to April 2012, participants aged 50-90 years with a diagnosis of clinically probable DLB were recruited from the behavioral neurology outpatient clinic and Alzheimer's Disease Research Center program at Mayo Clinic, Rochester, MN, USA, after a comprehensive neurobehavioral evaluation. We included patients with an ESS score of 8 or greater, with Folstein Mini-Mental State Examination scores between 10 and 26, and without any active medical disorder that could preclude participation in a drug treatment trial over a 12-week protocol. Participants were required to have had a stable medication regimen during the previous 4 weeks and could not be taking any psychotropic medication at doses viewed by the clinician to substantially affect the patient's alertness. For participant inclusion, we also desired a clinically significant primary sleep-related breathing disorder causing sleepiness to be either ruled out by polysomnography (PSG) or, if present, confirmed to be effectively treated by nasal continuous positive airway pressure (CPAP); exceptions were permitted in select circumstances if CPAP had been variably tolerated on prior PSG. An identified caregiver for at least $4 \mathrm{~h}$ per day for at least 5 days per week was required for participation in the study, and both patients and caregivers had to be willing and able to consent and participate in all study-related procedures. 
Dementia

and Geriatric Cognitive Disorders

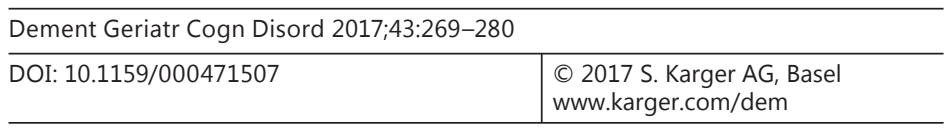

Lapid et al.: Efficacy, Safety, and Tolerability of Armodafinil Therapy for Hypersomnia Associated with Dementia with Lewy Bodies: A Pilot Study

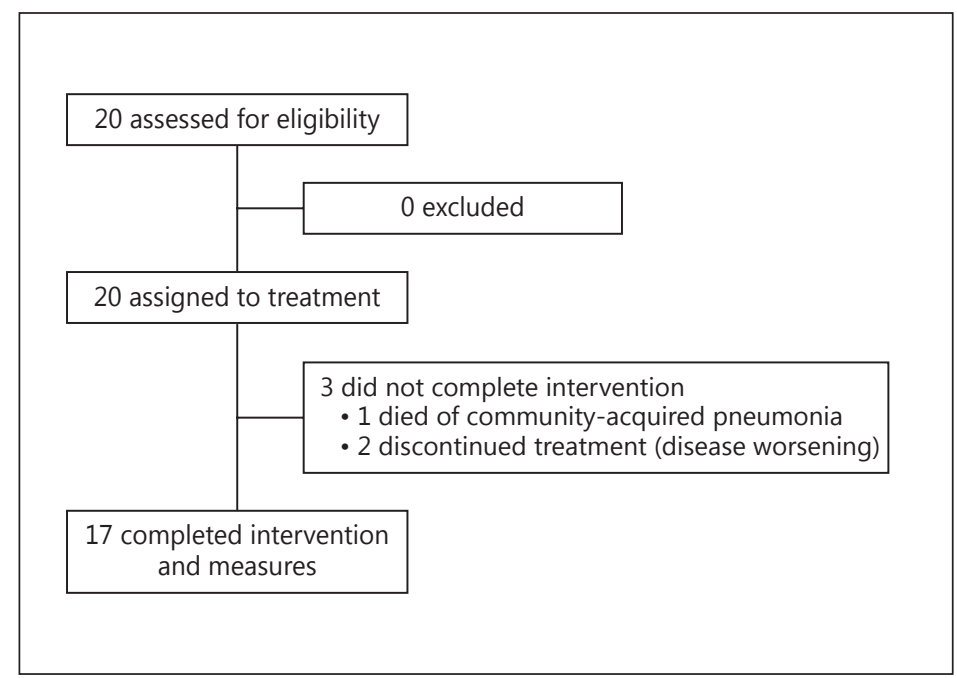

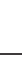

Fig. 1. CONSORT flow diagram of

participant enrollment.

participant enrollment. 
Lapid et al.: Efficacy, Safety, and Tolerability of Armodafinil Therapy for Hypersomnia Associated with Dementia with Lewy Bodies: A Pilot Study

Table 1. Patients' demographic and clinical data

\begin{tabular}{|c|c|c|c|c|c|c|c|}
\hline \multirow{2}{*}{$\begin{array}{l}\text { Patient No. / } \\
\text { sex/age, years }\end{array}$} & \multicolumn{4}{|c|}{ Age at onset, years } & \multirow[t]{2}{*}{$\mathrm{CDR}$} & \multirow[t]{2}{*}{ MMSE } & \multirow[t]{2}{*}{ Psychoactive medications } \\
\hline & RBD & $\operatorname{Cog}$ & Park & VH & & & \\
\hline $1 / \mathrm{M} / 66$ & 45 & 62 & 62 & 63 & 0.5 & 20 & Rivastigmine, memantine, citalopram \\
\hline $2 / F / 73$ & 64 & 67 & 71 & 72 & 1 & 24 & Donepezil, memantine \\
\hline $3 / F / 73$ & NA & 69 & 72 & 72 & 1 & 20 & Donepezil, memantine, mirtazapine \\
\hline $4 / \mathrm{M} / 66$ & 66 & 52 & 62 & NA & 1 & 22 & Donepezil, memantine, rasagiline \\
\hline $5 / \mathrm{M} / 70$ & 54 & 64 & 65 & NA & 1 & 23 & Donepezil, memantine, citalopram, melatonin \\
\hline $6 / F / 83$ & 76 & 72 & 82 & 76 & 2 & 13 & Galantamine, melatonin \\
\hline $7 / \mathrm{M} / 74$ & 72 & 68 & 70 & 72 & 1 & 26 & Donepezil, carb/L-dopa \\
\hline $8 / \mathrm{M} / 66$ & 61 & 57 & 61 & 61 & 2 & 14 & Donepezil, memantine \\
\hline $9 / \mathrm{M} / 68$ & 57 & 63 & 65 & 63 & 2 & 19 & Galantamine, melatonin, escitalopram \\
\hline $10 / \mathrm{M} / 68$ & 59 & 63 & 63 & 63 & 1 & 26 & Carb/L-dopa, fluoxetine, clonazepam \\
\hline $11 / \mathrm{M} / 52$ & 41 & 45 & 47 & 49 & 1 & 22 & Donepezil, memantine, sertraline, clonazepam \\
\hline $12 / \mathrm{M} / 67$ & 49 & 62 & 62 & 63 & 1 & 21 & Donepezil, sertraline, quetiapine \\
\hline $13 / M / 66$ & 50 & 51 & 51 & 63 & 1 & 26 & Rivastigmine, carb/L-dopa, pramipexole, clonazepam \\
\hline $14 / \mathrm{M} / 79$ & 72 & 73 & 75 & 74 & 1 & 18 & Rivastigmine, clonazepam, escitalopram \\
\hline $15 / \mathrm{M} / 80$ & 72 & 72 & 77 & NA & 1 & 17 & Galantamine, carb/L-dopa, clonazepam, citalopram \\
\hline $16 / \mathrm{M} / 69$ & 65 & 62 & 66 & 66 & 2 & 18 & Memantine \\
\hline $17 / F / 78$ & 77 & 74 & 77 & 76 & 1 & 24 & Rivastigmine, melatonin \\
\hline $18 / \mathrm{M} / 83$ & NA & 75 & 82 & NA & 0.5 & 22 & Donepezil, memantine \\
\hline $19 / \mathrm{M} / 82$ & 77 & 77 & 77 & NA & 2 & 23 & Donepezil \\
\hline $20 / M / 73$ & 50 & 64 & 70 & 72 & 1 & 26 & Galantamine, clonazepam, carb/L-dopa \\
\hline
\end{tabular}

carb/L-dopa, carbidopa/levodopa; CDR, global Clinical Dementia Rating scale score; Cog, cognitive decline; MMSE, MiniMental State Examination score; NA, not applicable; Park, parkinsonism; RBD, REM sleep behavior disorder; VH, visual hallucinations. ${ }^{\text {a }}$ All patients except patient 1,10 , and 12 completed the trial.

\section{Results}

\section{Patient Characteristics}

Of 20 participants screened, all were enrolled; 2 discontinued near completion of the study because of worsening disease, and 1 died of community-acquired pneumonia. Seventeen participants completed the 12-week protocol (Fig. 1). The patients' demographic and clinical data are shown in Table 1. The demographic characteristics for all 20 participants and caregivers are given in online supplemental Table 1 . The median age of all patients was 72 years; most were men (80\%), and almost all had spouses as caregivers.

All participants had baseline PSG; 4 were using CPAP. The mean respiratory disturbance index was 11.5; 5 patients had a value of 20 or greater. Four of these 5 had known obstructive sleep apnea (OSA) and had been intolerant to CPAP; in the other participant, the respiratory disturbance index on PSG was 23, despite use of CPAP, due to central apneic events.

\section{Efficacy}

The changes in scores from baseline to end of study for the ESS, MWT, ADCS-CGIC, ADASADL, ADAS-Cog, supplemental neuropsychological measures, NPI, and QOL are presented in Table 2. The measurements at baseline, week 4, week 8 , and week 12 are given in online supplemental Table 2 . Regarding the primary efficacy assessment, the ESS $(p<0.001)$ and MWT ( $p=0.003$ ) scores were improved at the end of the study compared with baseline (Fig. 2). For the ESS, we used a cutoff of 10 as reflecting excessive daytime somnolence; 18 of the 20 patients had scores above this cutoff at baseline, and 12 of these $18(67 \%)$ had ESS 
Table 2. Efficacy assessments from baseline to week 12

\begin{tabular}{|c|c|c|c|c|}
\hline \multirow[t]{2}{*}{ Characteristic } & \multicolumn{3}{|l|}{ Score $^{a}$} & \multirow[t]{2}{*}{$p$ value $^{\mathrm{b}}$} \\
\hline & baseline & week 12 & difference & \\
\hline \multicolumn{5}{|l|}{ Sleep measures } \\
\hline ESS & $12.5(8-23)$ & $6.5(1-18)$ & $6.5(-1$ to 15$)$ & $<0.001$ \\
\hline MWT & $6.7(1-24)$ & $19.0(2-40)(n=17)$ & $-6.2(-32$ to 4$)(n=17)$ & 0.003 \\
\hline \multicolumn{5}{|l|}{ Global/functional measures } \\
\hline ADCS-CGIC & - & $3.0(1-5)$ & - & $<0.001^{\mathrm{c}}$ \\
\hline ADAS-ADL & $54(14-74)$ & $52.5(12-74)$ & $0(-14$ to 16$)$ & 0.65 \\
\hline \multicolumn{5}{|l|}{ Cognitive measure } \\
\hline ADAS-Cog & $38.8(28-63)(n=18)$ & $38.3(22-60)(n=19)$ & $1.2(-11$ to 11$)(n=18)$ & 0.66 \\
\hline \multicolumn{5}{|c|}{ Supplemental neuropsychological measures } \\
\hline MMSE total score & $22(13-26)$ & $21(9-29)$ & $0.5(-6$ to 4$)$ & 0.79 \\
\hline Digit Span forward length & $6.0(3-8)$ & $5.0(3-7)$ & $1.0(-1$ to 3$)$ & 0.04 \\
\hline Digit Span backward length & $3.0(0-4)$ & $3.0(0-5)$ & $0(-3$ to 3$)$ & 0.72 \\
\hline COWAT & $17.0(1-49)$ & $15.0(4-51)$ & $-1.0(-11$ to 12$)$ & $>0.99$ \\
\hline Rey-Osterrieth, copy score & $5.8(1-22)$ & $5.2(0-20)$ & $1.2(-7$ to 7$)$ & 0.43 \\
\hline \multicolumn{5}{|l|}{ Neuropsychiatric measures } \\
\hline NPI total score & $11.0(1-40)$ & $6.5(0-47)$ & $-4.0(-20$ to 21$)$ & 0.07 \\
\hline Delusions & $0(0-8)$ & $0(0-8)$ & $0(-6$ to 6$)$ & 0.61 \\
\hline Hallucinations & $1.5(0-8)$ & $0.5(0-12)$ & $0(-5$ to 8$)$ & 0.29 \\
\hline Agitation & $1.0(0-8)$ & $0.5(0-6)$ & $0(-4$ to 6$)$ & 0.70 \\
\hline Depression & $1.0(0-8)$ & $0.5(0-12)$ & 0 ( -7 to 8$)$ & 0.65 \\
\hline Anxiety & $1.0(0-8)$ & $0(0-12)$ & $0(-2$ to 8$)$ & 0.30 \\
\hline Euphoria & $0(0-12)$ & $0(0-12)$ & $0(-3$ to 0$)$ & $>0.99$ \\
\hline Apathy & $5.0(0-12)$ & $1.5(0-12)$ & $-2.5(-8$ to 6$)$ & 0.01 \\
\hline \multicolumn{5}{|l|}{ QOL measures } \\
\hline \multicolumn{5}{|l|}{ Patient self-report subscales } \\
\hline Physical & $8.0(5-10)$ & $6.0(3-10)$ & $2.0(-2$ to 5$)$ & 0.003 \\
\hline Emotional & $8.0(4-10)$ & $6.0(3-10)$ & $1.0(-2$ to 4$)$ & 0.03 \\
\hline Spiritual & $6.5(2-10)$ & $5.5(1-10)$ & 0.5 ( -4 to 5$)$ & 0.27 \\
\hline Religion & $6.0(1-10)$ & $4.5(1-10)$ & $0(-4$ to 6$)$ & 0.49 \\
\hline Mental & $7.0(3-9)$ & $5.5(3-9)$ & $2.0(-3$ to 5$)$ & 0.02 \\
\hline Social & $8.0(4-10)$ & $5.0(3-10)$ & $1.5(-2$ to 5$)$ & 0.004 \\
\hline Pain frequency & $3.0(1-9)$ & $3.5(1-8)$ & $0(-5$ to 7$)$ & 0.60 \\
\hline Pain severity & $2.0(1-10)$ & $2.5(1-8)$ & 0 ( -6 to 9$)$ & 0.71 \\
\hline Coping & $8.0(4-10)$ & $7.0(4-10)$ & $0.5(-3$ to 5$)$ & 0.20 \\
\hline \multicolumn{5}{|l|}{ Overall QOL } \\
\hline Patient self-report & $7.0(4-10)$ & $7.5(4-10)$ & $0(-3$ to 3$)$ & 0.98 \\
\hline Caregiver on patient & $6.5(1-10)$ & $7.0(3-10)$ & $0(-5$ to 6$)$ & 0.49 \\
\hline \multicolumn{5}{|l|}{ Caregiver overall QOL } \\
\hline self-report & $7.0(3-10)$ & $8.0(4-10)$ & $-1.0(-5$ to 0$)$ & 0.004 \\
\hline
\end{tabular}

ADAS-ADL, Alzheimer's Disease Assessment Scale-Activities of Daily Living scale; ADAS-Cog, Alzheimer's Disease Assessment Scale-cognitive subscale; ADCS-CGIC, Alzheimer's Disease Cooperative Study-Clinical Global Impression of Change; COWAT, Controlled OralWord Association Test; ESS, Epworth Sleepiness Scale; MMSE, Mini-Mental State Examination; MWT, Maintenance of Wakefulness Test; NPI, Neuropsychiatric Inventory; QOL, quality of life. ${ }^{\text {a }}$ Values are median (range). ${ }^{\mathrm{b}}$ Wilcoxon signed-rank test for difference between baseline and week 12 scores. ${ }^{\mathrm{c}}$ Wilcoxon signed-rank test for median $=4$. 
Lapid et al.: Efficacy, Safety, and Tolerability of Armodafinil Therapy for Hypersomnia Associated with Dementia with Lewy Bodies: A Pilot Study

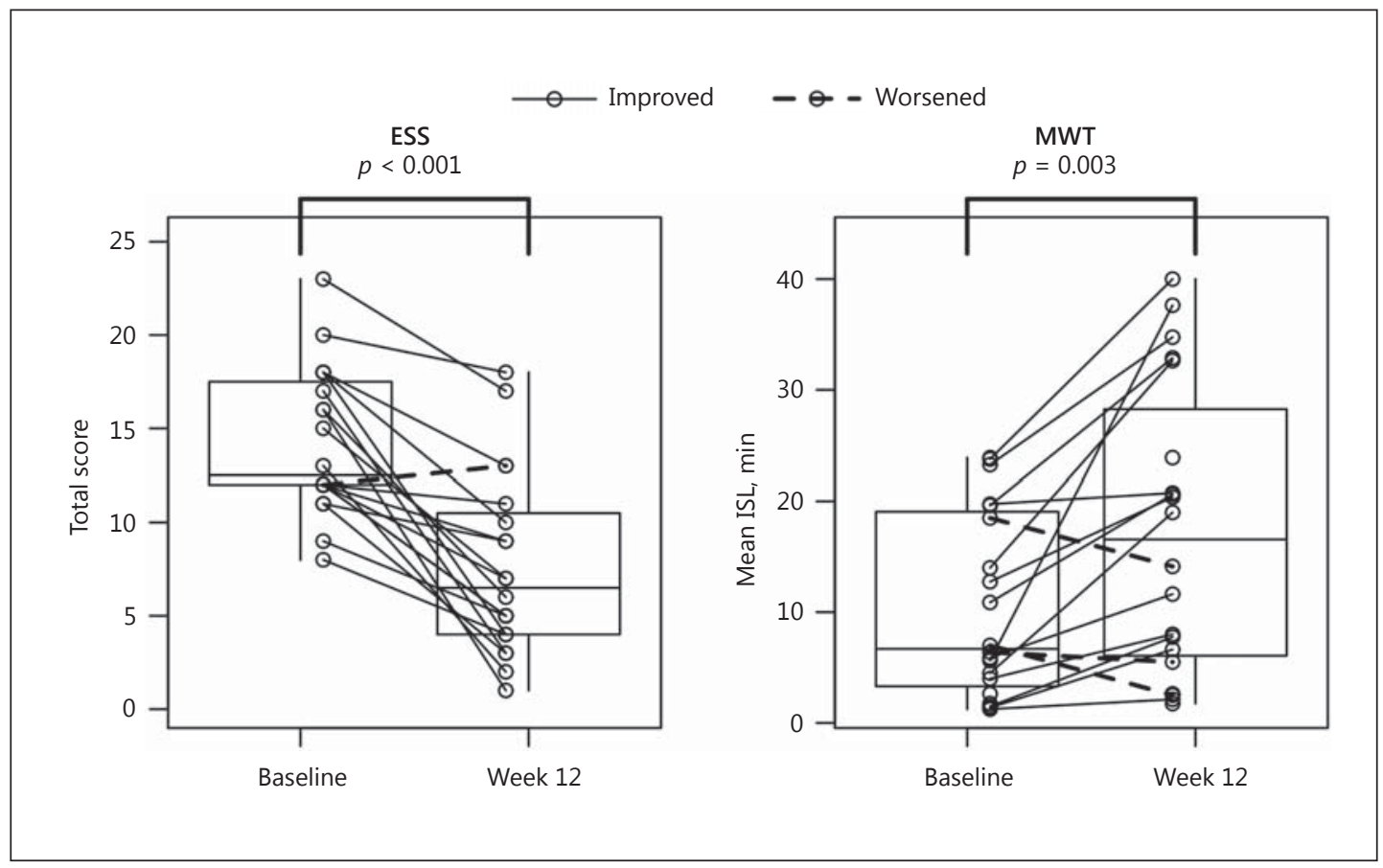

Fig. 2. Changes in daytime hypersomnolence with armodafinil. The box plots summarize the group scores on the Epworth Sleepiness Scale (ESS) and Maintenance of Wakefulness Test (MWT) at baseline and week 12 of armodafinil treatment; individual patient scores are also shown $(n=20)$. ISL, initial sleep latency.

scores of less than 10 at their final visit while on armodafinil therapy. The other 2 patients whose ESS score had been 8 at baseline had lower ESS values at the final visit. All but 1 participant experienced improvement on the ESS at their last observation compared with baseline. Using a cutoff of $8 \mathrm{~min}$ ( $2 \mathrm{SD}$ below the mean of $30 \mathrm{~min}$ based on published normative data) [19] on the 40-min version of the MWT, 11 patients (55\%) had mean initial sleep latency (ISL) values of 8 min or less at baseline, and $12(60 \%)$ had ISL values greater than 8 at their final visit while on armodafinil therapy. All but 3 participants had improved MWT scores at their last observation compared with baseline.

Regarding the secondary efficacy assessment, the ADCS-CGIC $(p<0.001)$ scores were improved at the end of the study, but significant changes from baseline were not found on the ADAS-ADL and ADAS-Cog measures over time. No improvement occurred on any of the cognitive measures, and worsening occurred on a few measures (e.g., Digit Span forward). The total NPI scores were significantly improved after 4 weeks of armodafinil therapy ( $p=$ 0.003); the median total scores were lower at the 8- and 12-week visits compared with baseline, but this did not reach significance. Specific neuropsychiatric features of visual hallucinations $(p=0.003)$ and agitation $(p=0.02)$ showed significant decreases after 4 weeks. The most notable improvement on the NPI was in apathy, with sustained improvement throughout the study: week 4 ( $p=0.02)$, week $8(p=0.008)$, and week 12 ( $p=0.01)$ (Fig. 3). There was no evidence of significant decreases or increases in delusions, depression, euphoria, or anxiety. Overall QOL improved for caregivers, with higher scores at the end of the study $(p=$ 0.004). There were no significant differences found in the overall QOL for patients, whether self-reported or rated by the caregiver. The patients rated the individual QOL domains of physical $(p=0.003)$, emotional $(p=0.03)$, mental $(p=0.02)$, and social $(p=0.004)$ well-being lower at week 12 than they did at baseline. 
Lapid et al.: Efficacy, Safety, and Tolerability of Armodafinil Therapy for Hypersomnia Associated with Dementia with Lewy Bodies: A Pilot Study

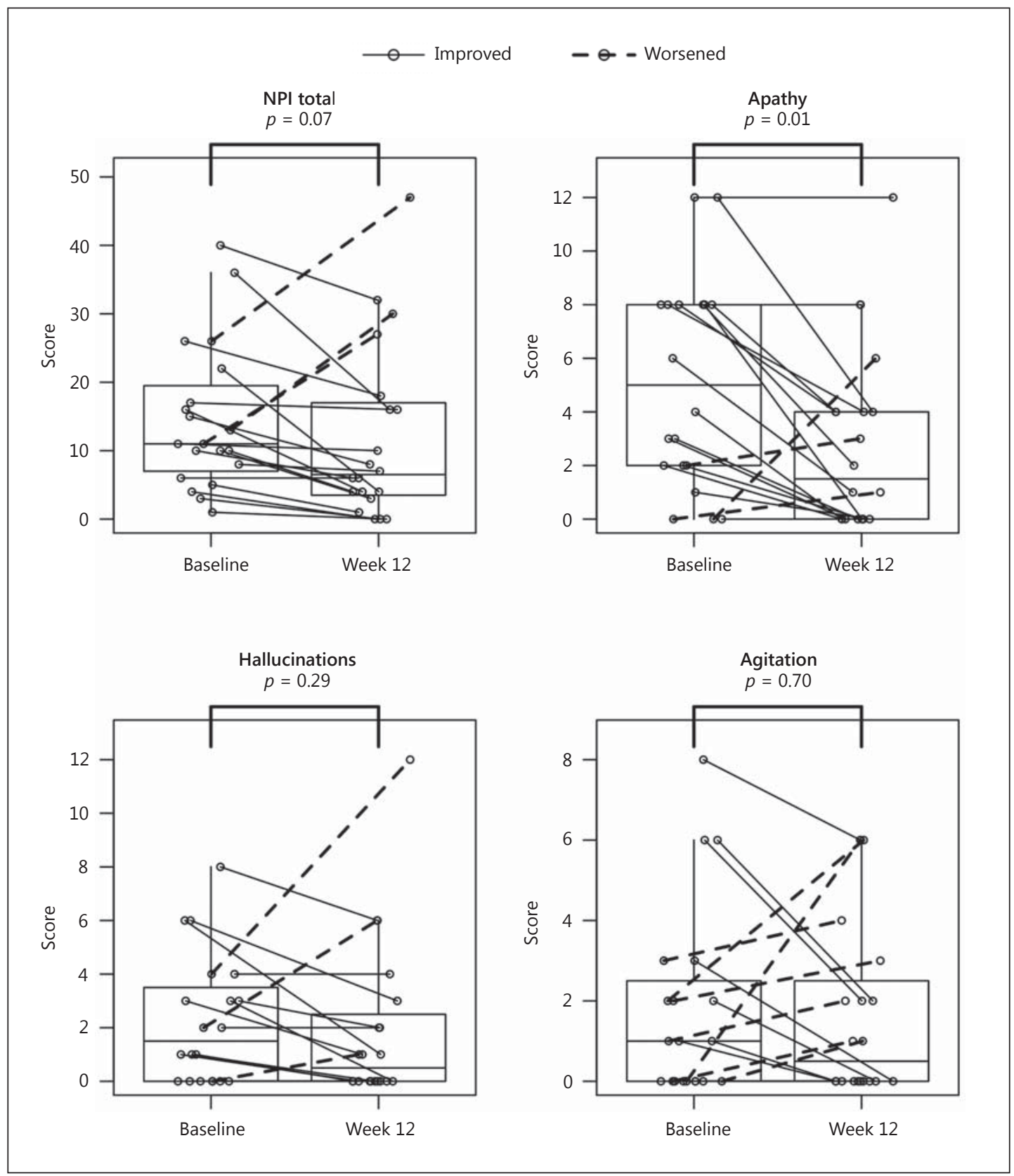

Fig. 3. Changes in Neuropsychiatric Inventory (NPI) scores with armodafinil. The box plots summarize the group scores on the NPI total, apathy, visual hallucination, and agitation scales at baseline and week 12 of armodafinil treatment; individual patient scores are also shown $(n=20)$.

\section{Safety and Tolerability}

All participants tolerated armodafinil dosing at $150 \mathrm{mg} /$ day during the baseline-to4-week phase. In 17 patients, the dosage was increased to $250 \mathrm{mg} /$ day, which was maintained throughout their participation in the protocol; the other 3 participants are described below. There were no clinically significant changes from baseline through the end of the study in laboratory parameters for renal function, liver function, complete blood count, electrolytes, and heart rate. No clinically significant adverse events were observed or reported. The 
median QTc intervals were $421.5 \mathrm{~ms}$ at baseline and $429 \mathrm{~ms}$ at the end of the study. There were no clinically significant differences in change of QTc interval from baseline, except for 1 participant. In this participant, the QTc increased from $395 \mathrm{~ms}$ at baseline to $439 \mathrm{~ms}$ at week 4; the dose of armodafinil was maintained at $150 \mathrm{mg} /$ day until repeat electrocardiography showed a decreased QTc, at which point the dosage was increased to $250 \mathrm{mg} /$ day and maintained. The QTc had returned to baseline level at the end of the study. Another participant had increased anxiety during the initial 4-week phase at $150 \mathrm{mg} /$ day, so armodafinil was discontinued for 2 weeks. The anxiety did not change, so the $150 \mathrm{mg} /$ day armodafinil regimen was resumed and then increased to $250 \mathrm{mg} /$ day. This participant did not complete the protocol because of social issues, and he could not return for further visits. For the other participant, the dosage was $150 \mathrm{mg} /$ day, then $250 \mathrm{mg} /$ day, then $150 \mathrm{mg} /$ day, and then $250 \mathrm{mg} /$ day (changing weekly) over the final 4 weeks of the study.

\section{Discussion}

Hypersomnia in DLB is common and debilitating, but it can be moderated with pharmacologic interventions in conjunction with behavioral management [1]. Our pilot data suggest improvements in caregiver-observed hypersomnia and objective wakefulness compared with baseline, along with reasonable safety and tolerability, with the use of armodafinil therapy in hypersomnolent patients with DLB. Prospective, randomized, double-blind studies in patients with DLB are warranted.

Our study findings suggest that armodafinil was effective in reducing daytime sleepiness and improving wakefulness over 12 weeks in our study population. Armodafinil is a wakefulness-promoting agent that is a levorotatory $(R)$ enantiomer of the racemic compound modafinil and is twice as potent $[20,21]$. In the USA, modafinil and armodafinil are approved for promoting wakefulness in patients with excessive sleepiness associated with OSA/ hypopnea syndrome, narcolepsy, and shift work sleep disorder [20,21]. The precise mechanisms through which armodafinil or modafinil promote wakefulness are unknown. Five randomized, double-blind, placebo-controlled, parallel-group studies demonstrated the efficacy and safety of 12-week armodafinil treatment in adults with excessive sleepiness associated with sleep apnea/hypopnea syndrome [22, 23], shift work sleep disorder [24], narcolepsy [15], or closed traumatic brain injury [25].

One randomized controlled trial demonstrated modafinil to be effective in treating daytime sleepiness [26], and 2 randomized controlled trials investigated the use of modafinil to treat fatigue $[27,28]$, all in patients with Parkinson disease. A review of efficacy data on the nonmotor symptoms of Parkinson disease found insufficient evidence regarding the efficacy of modafinil for the treatment of excessive daytime sleepiness, and of methylphenidate and modafinil for the treatment of fatigue [29]. A systematic review of 10 randomized controlled trials of modafinil found therapeutic effects for excessive daytime somnolence but not fatigue in patients with Parkinson disease, as well as inconsistent results on fatigue and excessive daytime somnolence in other neurologic disorders (multiple sclerosis, traumatic brain injury, and postpolio syndrome) [30]. The physiologic underpinnings of hypersomnia and fatigue may be different; fatigue was not investigated in our study.

Although many studies have examined the effects of stimulants and wake-promoting agents on sleep and wakefulness in these various other conditions, no formal prospective studies of wake-promoting agents have been performed in patients with DLB. To our knowledge, our study is the first to investigate the use of armodafinil to treat hypersomnolence in patients with DLB, and there are no comparable studies. It is important to note, however, that although $67 \%$ of the participants in our study had improvement in ESS scores 
from a pathologic range $(\geq 10)$ to a nonpathologic range $(<10)$ and although $20 \%$ had improvement in MWT ISL values from a pathologic range $(\leq 8 \mathrm{~min})$ to a nonpathologic range ( $>8 \mathrm{~min}$ ), there was still evidence of hypersomnia based on the ESS and MWT. Therefore, although armodafinil did improve hypersomnia in patients with DLB in this study, a substantial proportion continued to experience hypersomnia based on the ESS and/or MWT.

Our study patients had clinically significant improvement in global functioning at the end of the study, as assessed by clinicians on the ADCS-CGIC. However, no significant changes were observed for functional or cognitive status over 12 weeks of armodafinil treatment, as measured by the ADAS-ADL and ADAS-Cog, respectively. Several studies suggest not only improved cognitive performance but also improved psychomotor vigilance with the use of modafinil and armodafinil [31]. There is also evidence of improvement in the cognitive abilities of healthy patients and psychiatric populations taking modafinil [32]. One systematic review reported that psychostimulants had little or no effect on cognition in dementia, but this review did not distinguish true psychostimulants from wake-promoting agents [33]. A small study described improvement in attention and global mental status in a retrospective sample of 6 patients with Parkinson disease dementia/DLB receiving modafinil or armodafinil [34]. The study also reported better reaction times and accuracy to a visual stimulus on computerized attention tasks, reflecting changes in cognitive fluctuations, in a prospective sample of 7 patients with Parkinson disease dementia/DLB with an open-label single-dose study [34]. In our study participants, no changes in functional or cognitive status were detected over time, although a prospective study involving more patients using a similar battery of neuropsychological measures is still warranted.

Our findings on the secondary efficacy measures suggest improvement of overall neuropsychiatric morbidity, visual hallucinations, and agitation in the short term (4 weeks) and improvement of apathy sustained throughout the study (12 weeks). Caregivers endorsed a higher overall QOL at the end of the study, whereas overall QOL for the patients remained the same. The complex and multiple symptoms of motor and autonomic dysfunction, cognitive fluctuations, visual hallucinations and behavioral changes, and sleep-related issues in DLB place a high symptom burden on the patients and care challenges for the caregivers, which affects QOL for both groups. Patients with DLB have more frequent and clinically significant hallucinations and apathy than do patients with Alzheimer disease [35]. The factors most likely to contribute to caregiver burden were sleep and mood disturbances [36], and the higher distress that DLB caregivers experience is associated with neuropsychiatric symptoms of delusions, hallucinations, anxiety, and apathy [37, 38]. Other factors associated with caregiver burden include daytime sleepiness and mood disturbances [36, 39]. Our study findings of improved overall neuropsychiatric status and reduced hallucinations, agitation, and apathy may be related to the caregivers' perception that their QOL was better at the end of the study. An important clinical implication of this finding is that pharmacologic treatments that may improve neuropsychiatric symptoms in DLB have the potentially desirable consequence of improving the QOL of caregivers.

Our findings suggest the overall safety and tolerability of armodafinil in our patients. There were no abnormalities in laboratory test results, heart rate, or vital signs at the end of the study. We noted no worsening of parkinsonian symptoms or any adverse effects. Despite being similar to sympathomimetic amine stimulant agents such as amphetamine and methylphenidate in their wakefulness-promoting actions, armodafinil and modafinil are distinct from the stimulants in their adverse effect profile, including lack of hyperlocomotion, hyperthermia, rebound hypersomnolence, and minimal effects on cardiovascular and hemodynamic parameters [3]. Stimulants have also been noted for the potential for abuse and induction of psychosis and should be avoided in patients with cardiac risk factors [3]. Although neuropsychiatric features may worsen with stimulants, clinical experience suggests that this 
Lapid et al.: Efficacy, Safety, and Tolerability of Armodafinil Therapy for Hypersomnia Associated with Dementia with Lewy Bodies: A Pilot Study

is uncommon. A study that assessed the pharmacokinetics of armodafinil in 2 age groups demonstrated increased systemic exposure in the elderly (aged $>65$ years) compared with younger patients (aged 18-45 years), as measured by steady-state area under the curve (tau) and $\mathrm{C}_{\max }$ values. Although the elderly participants did not have more adverse events than the younger participants, and even though armodafinil was well tolerated by both age groups, the recommendation is to consider using lower doses to manage excessive sleepiness in older patients [40].

Limitations of our study include the open-label design and lack of comparison with a control group. In addition, concomitant use of cholinesterase inhibitors and psychoactive therapies could influence alertness, although our participants had been on stable doses for at least 4 weeks before the study. The small sample size also may limit the generalizability of our findings. Finally, some of the participants in this study had OSA; ideally, all participants should either have no clinically significant OSA or have OSA successfully treated with CPAP. We believe that the inclusion of patients with OSA is justified because it reflects the suboptimal tolerability of CPAP in some patients with DLB (and in many other patient populations) and because this was designed as a pilot study. A placebo-controlled, double-blind approach with minimal potential confounders would be desired in future studies of wake-promoting agents in DLB.

DLB is a progressive and ultimately fatal neurodegenerative disorder for which no therapy has been shown to alter the rate of progression. Yet, our study findings have relevant implications for clinical practice and provide insights for a therapy in DLB that has not been emphasized sufficiently. Key strategies in the management of DLB should include pharmacologic approaches that target excessive daytime sleepiness and neuropsychiatric symptoms, because these compromise the QOL of patients and caregivers. Interventions that help reduce the symptom burden, while also being safe and well tolerated, would be desirable in this patient population.

\section{Acknowledgments}

We thank the participants and their caregivers for participating in this protocol.

\section{Disclosure Statement}

M.I.L., K.M.K., S.S.M., J.A.A., E.S.L., L.A.A., W.K., and D.A.D. have no disclosures. B.F.B. has served as an investigator for clinical trials sponsored by Cephalon, Inc., GE Healthcare, FORUM Pharmaceuticals, and C2N Diagnostics. He receives royalties from the publication of a book entitled Behavioral Neurology of Dementia (Cambridge Medicine, 2009). He serves on the Scientific Advisory Board of the Tau Consortium. He has consulted for Isis Pharmaceuticals. He receives research support from the National Institutes of Health (U01 AG045390, U54 NS092089, P50 AG016574, U01 AG006786, R01 AG015866, R01 AG032306, and R01 AG041797) and the Mangurian Foundation.

\section{Funding Sources}

Cephalon, Inc., the Robert H. and Clarice Smith and Abigail Van Buren Alzheimer's Disease Research Program of the Mayo Foundation, National Institute on Aging grants AG015866 and AG016574, and the Mangurian Foundation. The staff at Cephalon, Inc., were not involved in the design and conduct of the study; in the collection, management, analysis, and interpretation of the data; or in the preparation, review, or approval of the manuscript. 
Dementia

\begin{tabular}{l|l}
\hline Dement Geriatr Cogn Disord 2017;43:269-280 \\
\hline DOI: 10.1159/000471507 & $\begin{array}{l}\text { C 2017 S. Karger AG, Basel } \\
\text { www.karger.com/dem }\end{array}$ \\
\hline
\end{tabular}

Lapid et al.: Efficacy, Safety, and Tolerability of Armodafinil Therapy for Hypersomnia Associated with Dementia with Lewy Bodies: A Pilot Study

\section{Author Contributions}

Study concept and design: B.F.B.; acquisition of data: K.M.K., L.A.A., D.A.D., and B.F.B.; analysis and interpretation of data: M.I.L., K.M.K., S.S.M., J.A.A., E.S.L., L.A.A., W.K., D.A.D., and B.F.B.; drafting of the manuscript: M.I.L., J.A.A., E.S.L., W.K., and B.F.B.; critical revision of the manuscript for important intellectual content: M.I.L., K.M.K., S.S.M., J.A.A., E.S.L., L.A.A., W.K., D.A.D., and B.F.B.; statistical analysis: J.A.A., E.S.L., and W.K.; obtained funding: B.F.B.; administrative, technical, and material support: K.M.K. and B.F.B.; study supervision: B.F.B.

\section{References}

1 McKeith IG, Dickson DW, Lowe J, Emre M, O’Brien JT, Feldman H, et al; Consortium on DLB: Diagnosis and management of dementia with Lewy bodies: third report of the DLB Consortium. Neurology 2005;65:18631872. Erratum in: Neurology 2005;65:1992.

2 Boeve BF: Parkinson-related dementias. Neurol Clin 2007;25:761-781, vii.

3 Boeve BF: Update on the diagnosis and management of sleep disturbances in dementia. Sleep Med Clin 2008; 3:347-360.

4 Ferman TJ, Boeve BF, Silber MH, Lin S-C, Tippmann-Peikert M, Smith GE: Daytime hypersomnolence on the multiple sleep latency test in patients with dementia with Lewy bodies. Alzheimers Dement 2008;4(suppl 4):T436.

5 Buracchio T, Arvanitakis Z, Gorbien M: Dementia with Lewy bodies: current concepts. Dement Geriatr Cogn Disord 2005;20:306-320.

6 Bliwise DL, Mercaldo ND, Avidan AY, Boeve BF, Greer SA, Kukull WA: Sleep disturbance in dementia with Lewy bodies and Alzheimer's disease: a multicenter analysis. Dement Geriatr Cogn Disord 2011;31:239-246.

7 Auning E, Rongve A, Fladby T, Booij J, Hortobágyi T, Siepel FJ, et al: Early and presenting symptoms of dementia with Lewy bodies. Dement Geriatr Cogn Disord 2011;32:202-208.

8 Wang HF, Yu JT, Tang SW, Jiang T, Tan CC, Meng XF, et al: Efficacy and safety of cholinesterase inhibitors and memantine in cognitive impairment in Parkinson's disease, Parkinson's disease dementia, and dementia with Lewy bodies: systematic review with meta-analysis and trial sequential analysis. J Neurol Neurosurg Psychiatry 2015;86:135-143.

9 Chwiszczuk L, Breitve M, Hynninen M, Gjerstad MD, Aarsland D, Rongve A: Higher frequency and complexity of sleep disturbances in dementia with Lewy bodies as compared to Alzheimer's disease. Neurodegener Dis 2016;16:152-160.

10 Ferman TJ, Smith GE, Dickson DW, Graff-Radford NR, Lin SC, Wszolek Z, et al: Abnormal daytime sleepiness in dementia with Lewy bodies compared to Alzheimer's disease using the multiple sleep latency test. Alzheimers Res Ther 2014;6:76.

11 Boeve BF: Clinical, diagnostic, genetic and management issues in dementia with Lewy bodies. Clin Sci (Lond) 2005;109:343-354.

12 Boeve BF: Dementia with Lewy bodies; in Schapira AH (ed): Neurology and Clinical Neuroscience. Philadelphia, Elsevier, 2007, pp 911-926.

13 Ferman TJ, Boeve BF: Dementia with Lewy bodies. Neurol Clin 2007;25:741-760.

14 Boeve BF, Silber MH, Saper CB, Ferman TJ, Dickson DW, Parisi JE, et al: Pathophysiology of REM sleep behaviour disorder and relevance to neurodegenerative disease. Brain 2007;130:2770-2788.

15 Harsh JR, Hayduk R, Rosenberg R, Wesnes KA, Walsh JK, Arora S, et al: The efficacy and safety of armodafinil as treatment for adults with excessive sleepiness associated with narcolepsy. Curr Med Res Opin 2006;22: 761-774.

16 Krahn LE, Hershner S, Loeding LD, Maski KP, Rifkin DI, Selim B, et al: Quality measures for the care of patients with narcolepsy. J Clin Sleep Med 2015;11:335.

17 Morgenthaler TI, Kapur VK, Brown T, Swick TJ, Alessi C, Aurora RN, et al; Standards of Practice Committee of the American Academy of Sleep Medicine: Practice parameters for the treatment of narcolepsy and other hypersomnias of central origin. Sleep 2007;30:1705-1711. Erratum in: Sleep 2008;31:table of contents.

18 Schwartz JR, Khan A, McCall WV, Weintraub J, Tiller J: Tolerability and efficacy of armodafinil in naïve patients with excessive sleepiness associated with obstructive sleep apnea, shift work disorder, or narcolepsy: a 12-month, open-label, flexible-dose study with an extension period. J Clin Sleep Med 2010;6:450-457.

19 Doghramji K, Mitler MM, Sangal RB, Shapiro C, Taylor S, Walsleben J, et al: A normative study of the maintenance of wakefulness test (MWT). Electroencephalogr Clin Neurophysiol 1997;103:554-562.

20 Armodafinil (CEP-10953). Investigator's brochure, ed 5. Release date May 20, 2008. Cephalon, Inc.

21 Nuvigil (armodafinil) tablets. Package insert. July 2008. Cephalon, Inc.

22 Hirshkowitz M, Black JE, Wesnes K, Niebler G, Arora S, Roth T: Adjunct armodafinil improves wakefulness and memory in obstructive sleep apnea/hypopnea syndrome. Respir Med 2007;101:616-627. 
Lapid et al.: Efficacy, Safety, and Tolerability of Armodafinil Therapy for Hypersomnia Associated with Dementia with Lewy Bodies: A Pilot Study

23 Roth T, White D, Schmidt-Nowara W, Wesnes KA, Niebler G, Arora S, et al: Effects of armodafinil in the treatment of residual excessive sleepiness associated with obstructive sleep apnea/hypopnea syndrome: a 12-week, multicenter, double-blind, randomized, placebo-controlled study in nCPAP-adherent adults. Clin Ther 2006;28:689-706.

24 Czeisler CA, Walsh JK, Wesnes KA, Arora S, Roth T: Armodafinil for treatment of excessive sleepiness associated with shift work disorder: a randomized controlled study. Mayo Clin Proc 2009;84:958-972.

25 Menn SJ, Yang R, Lankford A: Armodafinil for the treatment of excessive sleepiness associated with mild or moderate closed traumatic brain injury: a 12-week, randomized, double-blind study followed by a 12-month open-label extension. J Clin Sleep Med 2014;10:1181-1191.

26 Högl B, Saletu M, Brandauer E, Glatzl S, Frauscher B, Seppi K, et al: Modafinil for the treatment of daytime sleepiness in Parkinson's disease: a double-blind, randomized, crossover, placebo-controlled polygraphic trial. Sleep 2002;25:905-909.

27 Lou JS, Dimitrova DM, Park BS, Johnson SC, Eaton R, Arnold G, et al: Using modafinil to treat fatigue in Parkinson disease: a double-blind, placebo-controlled pilot study. Clin Neuropharmacol 2009;32:305-310.

28 Tyne HL, Taylor J, Baker GA, Steiger MJ: Modafinil for Parkinson's disease fatigue. J Neurol 2010;257:452-456.

29 Seppi K, Weintraub D, Coelho M, Perez-Lloret S, Fox SH, Katzenschlager R, et al: The Movement Disorder Society evidence-based medicine review update: treatments for the non-motor symptoms of Parkinson's disease. Mov Disord 2011;26(suppl 3):S42-S80.

30 Sheng P, Hou L, Wang X, Wang X, Huang C, Yu M, et al: Efficacy of modafinil on fatigue and excessive daytime sleepiness associated with neurological disorders: a systematic review and meta-analysis. PLoS One 2013; 8:e81802.

31 Edgar CJ, Pace-Schott EF, Wesnes KA: Approaches to measuring the effects of wake-promoting drugs: a focus on cognitive function. Hum Psychopharmacol 2009;24:371-389.

32 Minzenberg MJ, Carter CS: Modafinil: a review of neurochemical actions and effects on cognition. Neuropsychopharmacology 2008;33:1477-1502.

33 Dolder CR, Davis LN, McKinsey J: Use of psychostimulants in patients with dementia. Ann Pharmacother 2010; 44:1624-1632.

34 Varanese S, Perfetti B, Gilbert-Wolf R, Thomas A, Onofrj M, Di Rocco A: Modafinil and armodafinil improve attention and global mental status in Lewy bodies disorders: preliminary evidence. Int J Geriatr Psychiatry 2013;28:1095-1097.

35 Bjoerke-Bertheussen J, Ehrt U, Rongve A, Ballard C, Aarsland D: Neuropsychiatric symptoms in mild dementia with Lewy bodies and Alzheimer's disease. Dement Geriatr Cogn Disord 2012;34:1-6.

36 Galvin JE, Duda JE, Kaufer DI, Lippa CF, Taylor A, Zarit SH: Lewy body dementia: caregiver burden and unmet needs. Alzheimer Dis Assoc Disord 2010;24:177-181.

37 Ricci M, Guidoni SV, Sepe-Monti M, Bomboi G, Antonini G, Blundo C, et al: Clinical findings, functional abilities and caregiver distress in the early stage of dementia with Lewy bodies (DLB) and Alzheimer's disease (AD). Arch Gerontol Geriatr 2009;49:e101-e104.

38 Svendsboe E, Terum T, Testad I, Aarsland D, Ulstein I, Corbett A, et al: Caregiver burden in family carers of people with dementia with Lewy bodies and Alzheimer's disease. Int J Geriatr Psychiatry 2016;31:1075-1083.

39 Lee DR, McKeith I, Mosimann U, Ghosh-Nodyal A, Thomas AJ: Examining carer stress in dementia: the role of subtype diagnosis and neuropsychiatric symptoms. Int J Geriatr Psychiatry 2013;28:135-141.

40 Darwish M, Kirby M, Hellriegel ET, Yang R, Robertson P Jr: Systemic exposure to armodafinil and its tolerability in healthy elderly versus young men: an open-label, multiple-dose, parallel-group study. Drugs Aging 2011;28:139-150. 